\title{
NLO Electroweak Corrections to Higgs Boson Production at Hadron Colliders
}

\author{
Sandro Uccirati ${ }^{* \dagger}$ \\ Institut für Theoretische Teilchenphysik, Universität Karlsruhe, \\ 76128 Karlsruhe, Germany \\ E-mail: ucciratieparticle.uni-karlsruhe.de
}

\begin{abstract}
We present the complete two-loop electroweak corrections to $g g \rightarrow H$, the main partonic channel for the production of the Higgs boson at hadron colliders. The evaluation is based on a numerical approach and the result covers all kinematical regions, including $W W$ and $Z Z$ thresholds, by introducing complex masses for the $W$ and the $Z$ bosons. Furthermore the relation between physical observables measured at LHC and Tevatron and Higgs pseudo-observables (production cross section and partial decay widths) is revised by extensively using the notion of the Higgs complex pole. Numerical effects for the Higgs production cross section are presented.
\end{abstract}

RADCOR 2009 - 9th International Symposium on Radiative Corrections (Applications of Quantum Field Theory to Phenomenology),

October 25 - 302009

Ascona, Switzerland

\footnotetext{
${ }^{*}$ Speaker.

${ }^{\dagger}$ In collaboration with S.Actis, G.Passarino, C.Sturm
} 


\section{Introduction}

Gluon fusion is the main production channel of the SM Higgs-boson at hadron colliders, and both virtual and real corrections have been thoroughly investigated since the beginning of the 90's.

The QCD next-to-leading order (NLO) radiative corrections to the total Higgs-production cross section have been first computed below the $t \bar{t}$ threshold in Ref. [1], and using an effectiveLagrangian approach, where the top quark is integrated out, in Ref. [2]. Formally, the second method defines the heavy-top limit, and it has represented the starting point for subsequent higher order improvements. The result for the entire Higgs-mass range at NLO was derived in Ref. [3]. Since QCD NLO corrections increase the cross section by more than $70 \%$, there was a flurry of activity on higher order QCD effects. The next-to-next-to-leading order (NNLO) corrections to the total cross section have been calculated just in the heavy-top limit: partial results have been obtained in Ref. [4] and the complete NNLO cross section has been derived in Ref. [5]. An allorder resummed calculation of multiple soft-gluon emission at next-to-next-to-leading logarithmic (NNLL) accuracy has been performed in Ref. [6] and including higher logarithmic orders in Ref. [7]. Improvements with respect to the heavy-top limit have been recently obtained by the authors of Ref. [8].

The electroweak NLO corrections to the total cross section were evaluated in Ref. [9] in the heavy-top limit, and turned out to be less than $1 \%$. The contribution due to the light fermions has been calculated analytically in Ref. [10], and found to be more sizable; the remaining component of the amplitude involving the top quark has been computed by means of a Taylor expansion in the Higgs external momentum in Ref. [11] and is, for obvious reasons, valid in the kinematical region below the $W W$ threshold. The complete electroweak corrections were computed in Ref. [12] and are the topics of this article. Under the hypothesis of factorization with respect to the dominant QCD soft and collinear radiation, the impact of the EW corrections to Higgs-production in protonproton collisions has been estimated in Ref. [13]. The factorization hypothesis can be verified just by the direct computation of the $\mathrm{EW} \times \mathrm{QCD}$ corrections. The contribution to these corrections coming from top-loops was evaluated in Ref. [14] in the heavy-top limit, while the authors of Ref. [15] used an effective theory, valid in the small Higgs mass range, to compute the corrections arising from light quarks, finding agreement with the factorization hypothesis.

\section{Computation of the EW corrections}

The main problem that one encounters in the computation of electroweak processes at twoloop level is connected to the presence of many different mass scales. The common approach, which aims to write two-loop amplitudes in terms of analytic functions, relies on expansions in some of the scales of the problem restricting however the validity of the result to some regions of the parametric space. In order to have a result valid everywhere, we have abandoned the analytic way for a numerical evaluation of Feynman diagrams. In the numerical approach the (many) heavy mass scales can be easily treated, but a special care has to be devoted to light particles. Their small masses are natural regulators for the collinear singularities, but generate instabilities in the numerical integration. Therefore we have developed some techniques to extract explicitly the divergent behavior in these small masses, which are of logarithmic type. In the production process 
$g g \rightarrow H$ the total amplitude is free from collinear divergences and we could verify analytically that the logarithms in the small masses cancel when the contributions of all diagrams are summed up.

The strategy to obtain stable integral representations starts with Feynman parametrization. After the extraction of possible collinear logarithms the integrand always takes the form:

$$
z_{1}^{n_{1}} \cdots z_{k}^{n_{k}} V^{\mu}\left(z_{1}, \ldots, z_{k}\right) \ln ^{m} V\left(z_{1}, \ldots, z_{k}\right), \quad \mu=-1,-2,
$$

where $z_{1}, \ldots, z_{k}$ are the Feynman variables, integrated in some finite domain $\left(\subseteq[0,1]^{k}\right)$ and $n_{1}, \ldots, n_{k}$ are natural numbers. The function $V$ is quadratic with respect to a subset $\left\{z_{i}\right\}$ of $\left\{z_{1}, \ldots, z_{k}\right\}$ and each $z_{i}^{2}$ is proportional to one external squared momentum. This is a general feature of two-loop diagrams. The idea is to manipulate the integral and put it in the following form:

$$
\int d z \frac{Q(z)}{U(z)} f\left(\frac{U(z)}{P(z)}\right)
$$

where the function $f$ must satisfy $f(0)=0$ (possible candidates are the Spence functions $\ln ^{n}(1+x)$, $\left.\operatorname{Li}_{n}(x), \mathrm{S}_{n, p}(x)\right)$ and $Q, U$ and $P$ are polynomials. In this way the possible zeros of the denominator $U$ are compensated by the "regulating function" $f$ and the integral has no unstable points anymore.

In the case where the quadratic is not complete (i.e. some external squared momenta vanish) the solution is simple. For the process $g g \rightarrow H$ the two external squared momenta corresponding to the two gluons are always zero and therefore the quadratic $V$ can be written as $V=a x y+b x+$ $c y+d$, where $x, y$ are two integration variables and the parameters $a, b, c, d$ depend on the other variables and on the masses and momenta. For $\mu=-1,-2$ and $m=0$ we can use the following relations (similar relations hold for $m>0$ ):

$$
\begin{aligned}
\frac{1}{A x+B} & =\partial_{x} \frac{1}{A} \ln \left(1+\frac{A}{B} x\right) \quad A=a y+b, \quad B=c y+d \\
\frac{1}{(a x y+b x+c y+d)^{2}} & =-\partial_{x} \partial_{y} \frac{1}{a d-b c} \ln \left\{1+\frac{(a d-b c) x}{b(a x y+b x+c y+d)}\right\} .
\end{aligned}
$$

The two right-hand sides are exactly in the desired form: the zeros of the denominator correspond to the zeros of the logarithms. After an integration by parts to get rid of the derivatives, the result is ready to be integrated numerically.

\section{Behavior around $W W$ and $Z Z$ thresholds}

As explained in details in Refs. $[16,12]$ the two-loop amplitude for the partonic process $g g \rightarrow$ $H$ presents a singular behavior for $M_{H}=2 M_{W}$ and $M_{H}=2 M_{Z}$. This singularity is of squared root type and is represented by terms proportional to the inverse power of the threshold factor $\beta_{i}$,

$$
\beta_{i}=\sqrt{1-4 M_{i}^{2} / M_{H}^{2}}, \quad \text { with } \quad M_{i}=M_{W}, M_{Z} .
$$

The origin of this singularity is the derivative of the Higgs one-loop self-energy, associated with the Higgs wave function renormalization, as depicted in Fig. 1

$(1-$ loop diagrams $) \otimes(\mathrm{H}$ wave-function FR $)$

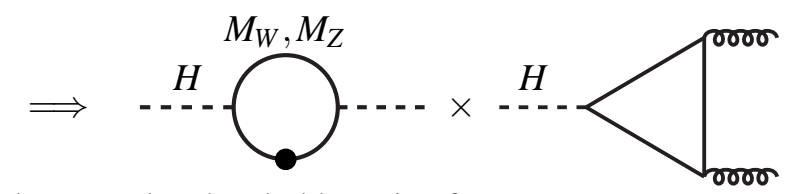

Figure 1: Singular $\beta^{-1}$ behavior at the normal $m$ threshold coming from WFR. 
A pragmatic gauge-invariant solution to the problem of threshold singularities due to unstable particles has been introduced and formalized in Ref. [17] and is termed in the following as minimal complex-mass (MCM) scheme. In this scheme the NLO amplitude is decomposed according to

$$
\mathscr{A}^{\mathrm{NLO}}=\sum_{i=W, Z} \frac{A_{\mathrm{SR}, i}}{\beta_{i}}+A_{\mathrm{REM}},
$$

where square-root-singular terms $\left(A_{\mathrm{SR}, i}\right)$ have been isolated from the component which is finite for $\beta_{i} \rightarrow 0\left(A_{\mathrm{REM}}\right)$. After proving that all coefficients in Eq.(3.2) satisfy separately the Ward identities, we minimally modify the amplitude introducing the complex-mass scheme of Ref. [18] for the divergent terms: the real masses of the $W$ and the $Z$ bosons are replaced by the corresponding complex poles in the threshold factors $\beta_{i}, i=W, Z$ and in the coefficients $A_{\mathrm{SR}, i}$ and the real parts of the $W$ and the $Z$ self-energies stemming from mass renormalization at one loop are traded for the complete self-energies, including imaginary parts.

The MCM scheme allows for a straightforward removal of unphysical infinities, but it does not deal with the artificial cusps associated with the crossing of normal thresholds, as shown in Fig. 2, which represent a concrete problem in assessing the impact of two-loop electroweak corrections. We have therefore undertaken the task of introducing the complete complex-mass (CM) scheme of Ref. [18], where the procedure described for the divergent terms of Eq.(3.2) has been extended to the remainder $A_{\text {REM }}$. In Fig. 2 are shown the two-loop electroweak percentage corrections for the partonic cross section $g g \rightarrow H$. The big contribution arising at large $M_{H}$ is basically due to the scalar sector of the SM, which is approaching the strong regime. This is a two-loop feature, being the one loop notoriously protected by Veltman's theorem.
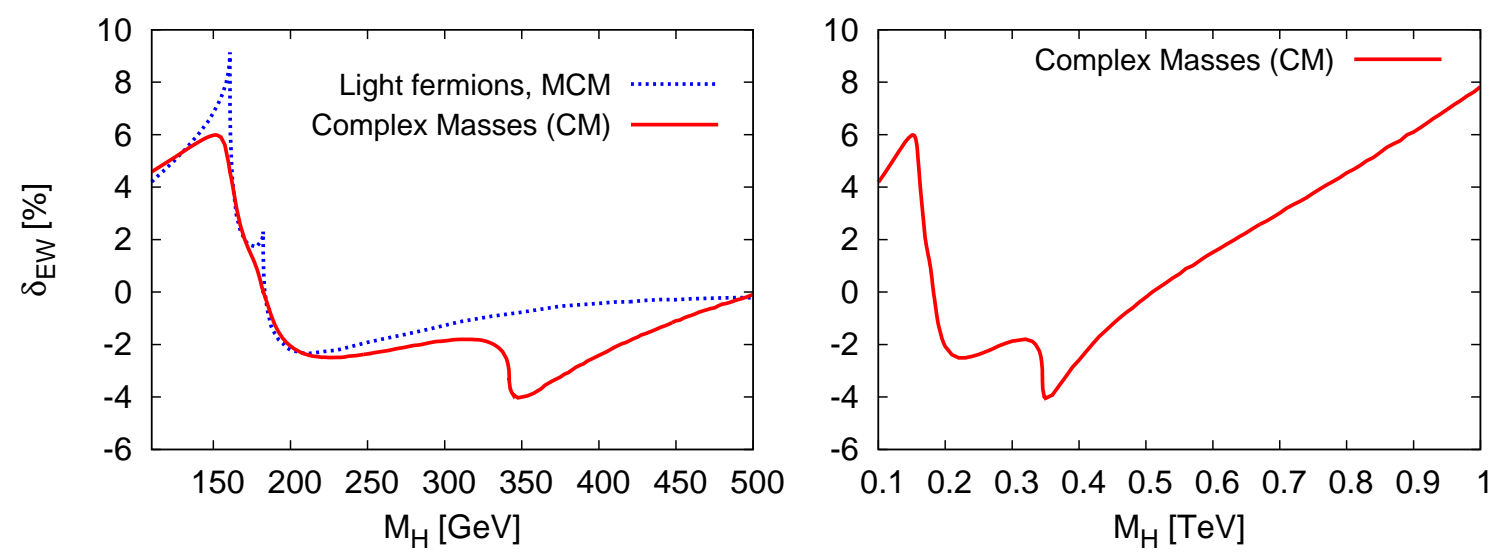

Figure 2: Two-loop electroweak percentage corrections for the partonic cross section $g g \rightarrow H$. The solid line denotes the total electroweak corrections in the MC scheme, including also top quarks, while the dotted line denotes the contribution coming from light fermion loops in the MCM scheme.

\section{Unstable particles and gauge invariance}

As pointed out in Ref. [17], the doubly-contracted Ward identity for the amplitude of the decay $H \rightarrow \gamma \gamma$ is not satisfied at two-loop level above the $W W$ threshold: the on-shell mass renormalization of the Higgs boson introduces the real part of the one-loop self-energy which does not cancel with the corresponding term coming from the pure loop part, containing the full self-energy. Gauge invariance is then violated unless one restores it by hand. The origin of the problem is connected to the common definition of production cross section and decay width, which treats the Higgs boson 
as an asymptotic state. But the Higgs boson is an unstable particle and should be removed from in/out bases in the Hilbert space and therefore, concepts as production of the Higgs boson or its partial decay widths do not have a precise meaning and should be replaced by conventionalized definitions which respect first principles of quantum field theory.

The quest for a proper treatment of unstable particles dates back to the sixties and to the work of Veltman [19] (for earlier attempts see Ref. [20]); more recently the question has been readdressed by Sirlin and collaborators [21]. The staring point is the concept of complex pole. The Higgs boson complex pole $\left(s_{H}\right)$ is the solution of the equation

$$
s_{H}-M_{H}^{2}+\Sigma_{H H}\left(s_{H}\right)=0,
$$

where $M_{H}^{2}$ is the lagrangian Higgs boson mass, real by construction, and $\Sigma_{H H}\left(s_{H}\right)$ is the Higgs selfenergy evaluated at $s=s_{H}$. This definition is gauge invariant, while the usual on-shell real Higgs mass is not. The Dyson re-summed Higgs propagator is given by

$$
\Delta_{H}(s)=\left(s-s_{H}\right)^{-1}\left[1+\Pi_{H H}(s)\right]^{-1}, \quad Z_{H}=1+\Pi_{H H}, \quad \Pi_{H H}(s)=\frac{\Sigma_{H H}(s)-\Sigma_{H H}\left(s_{H}\right)}{s-s_{H}} .
$$

At the parton level the $S$-matrix for a process $i \rightarrow f$ can be written as

$$
S_{f i}=V_{i}(s) \Delta_{H}(s) V_{f}(s)+B_{i f}(s),
$$

where $V_{i}$ is the production vertex $i \rightarrow H$ (e.g. $g g \rightarrow H$ ), $V_{f}$ is the decay vertex $H \rightarrow f$ (e.g. $H \rightarrow \gamma \gamma$ ) and $B_{i f}$ is the non-resonant background (e.g. $g g \rightarrow \gamma \gamma$ boxes). Using Eq.(4.2) we can write

$$
S_{f i}=\left[Z_{H}^{-\frac{1}{2}}(s) V_{i}(s)\right] \frac{1}{s-s_{H}}\left[Z_{H}^{-\frac{1}{2}}(s) V_{f}(s)\right]+B_{i f}(s)=\frac{S\left(i \rightarrow H_{c}\right) S\left(H_{c} \rightarrow f\right)}{s-s_{H}}+\underset{\substack{\text { non resonant } \\ \text { terms }}}{\text { t }}
$$

where we have extracted the relevant pseudo-observable,

$$
S\left(H_{c} \rightarrow f\right)=Z_{H}^{-1 / 2}\left(s_{H}\right) V_{f}\left(s_{H}\right),
$$

which is gauge independent by construction. A partial decay width can then be defined as

$$
\mu_{H} \Gamma\left(H_{c} \rightarrow f\right)=\frac{(2 \pi)^{4}}{2} \int d \Phi_{f}\left(P_{H},\left\{p_{f}\right\}\right) \sum_{\text {spins }}\left|S\left(H_{c} \rightarrow f\right)\right|^{2},
$$

where the integration is over the phase space spanned by $|f\rangle$, with the constraint $P_{H}=\sum p_{f}$. Similarly one can define a production cross section using the pseudo-observable $S\left(i \rightarrow H_{c}\right)$.

In order to compute these pseudo-observables we rely on the guiding principle that Green functions involving unstable particles should smoothly approach the value for stable ones (the usual Feynman $-i 0$ prescription) when the couplings of the theory (and therefore the imaginary parts of masses and momenta) tend to zero. To illustrate the procedure we consider the following one-loop scalar two-point function with complex masses and momenta:

$$
\text { m }=\Delta-\int_{0}^{1} d x \ln \chi, \quad \chi=-s_{H} x(1-x)+m^{2}, \quad s_{H}=M_{H}^{2}-i \Gamma_{H} M_{H}, \quad m^{2}=\mu^{2}-i \gamma \mu .
$$

Since both $s_{H}$ and $m^{2}$ are complex it can happen that $\chi$ is in the second quadrant, i.e. Re $\chi<0$ and $\operatorname{Im} \chi>0$. In this case (and just in this case) our guiding principle is violated:

$$
\lim _{\gamma, \Gamma_{H} \rightarrow 0} \operatorname{Im}[\ln \chi]=\pi \quad \neq \quad \begin{aligned}
& \text { Feynman prescription for real masses } \\
& \left(\text { i.e. } \gamma, \Gamma_{H}=0, \mu^{2} \rightarrow \mu^{2}-i 0\right)
\end{aligned}=-\pi
$$


It is important to notice that this can happen only with complex momenta (i.e. complex $s_{H}$ ): if just masses are complex, the imaginary part of the argument of the logarithm is always negative. In order to restore the proper real limit, we define an analytical continuation of the logarithm on the second Riemann sheet, by adding $-2 i \pi$ to it when $\chi$ is in the second quadrant. In general let us consider $\ln (z)$, where $z$ is a complex quantity which in the real mass limit gets an imaginary part of $\pm i 0$ from the Feynman prescription. The proper analytical continuation of $\ln (z)$ is then given by:

$$
\ln (z) \quad \rightarrow \quad \ln ^{ \pm}(z)=\ln (z) \pm 2 i \pi \theta(-\operatorname{Re} z) \theta(\mp \operatorname{Im} z)
$$

This is equivalent to moving the cut of the logarithm from the negative real axis to the positive (for $\ln ^{-}$) or negative (for $\ln ^{+}$) imaginary axis. If this is done at the integrand level (as in the present example) one has to take care that the integration contour does not cross the new cut. In that case a contour deformation in the complex $x$ plane has to be performed in order to get the correct result.

Taking into consideration all these technical aspects we were able to estimate the gauge invariant production cross section to $g g \rightarrow H$ at one-loop level and compare it with the usual one in the complex mass scheme. The effect, given in Fig. 3, is negligible for a Higgs mass up to the $t \bar{t}$-threshold, but becomes large for an heavy Higgs (of the order of QCD corrections), making questionable the use of a perturbative description of the Higgs-resonant part for $p p \rightarrow H$.

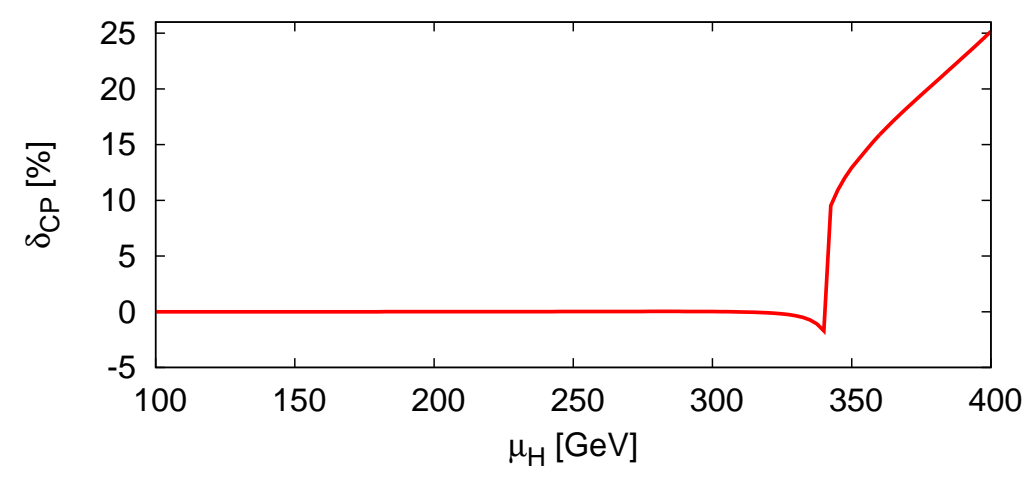

Figure 3: Comparison of the common production cross section $\sigma(g g \rightarrow H)$ with the gauge invariant pseudo-observable $\sigma_{C P}(g g \rightarrow H)$ based on the Higgs complex pole $s_{H}: \delta_{C P}=\sigma_{C P} / \sigma-1$.

\section{References}

[1] A. Djouadi, M. Spira and P.M. Zerwas, Phys. Lett. B 264 (1991) 440.

[2] S. Dawson, Nucl. Phys. B 359 (1991) 283.

[3] D. Graudenz, M. Spira and P.M. Zerwas, Phys. Rev. Lett. 70 (1993) 1372; M. Spira, A. Djouadi, D. Graudenz and P.M. Zerwas, Nucl. Phys. B 453 (1995) 17; R. Harlander and P. Kant, JHEP 0512 (2005) 015; C. Anastasiou, S. Beerli, S. Bucherer, A. Daleo and Z. Kunszt, JHEP 0701 (2007) 082; U. Aglietti, R. Bonciani, G. Degrassi and A. Vicini, JHEP 0701 (2007) 021; M. Muhlleitner and M. Spira, Nucl. Phys. B 790 (2008) 1.

[4] R.V. Harlander, Phys. Lett. B 492 (2000) 74; S. Catani, D. de Florian and M. Grazzini, JHEP 0105 (2001) 025; R.V. Harlander and W.B. Kilgore, Phys. Rev. D 64 (2001) 013015.

[5] R.V. Harlander and W.B. Kilgore, Phys. Rev. Lett. 88 (2002) 201801; C. Anastasiou and K. Melnikov, Nucl. Phys. B 646 (2002) 220; V. Ravindran, J. Smith and W.L. van Neerven, Nucl. Phys. B 665 (2003) 325; Nucl. Phys. Proc. Suppl. 135 (2004) 35.

[6] S. Catani, D. de Florian, M. Grazzini and P. Nason, JHEP 0307 (2003) 028. 
[7] S.O. Moch and A. Vogt, Phys. Lett. B 631 (2005) 48. E. Laenen and L. Magnea, Phys. Lett. B 632 (2006) 270; A. Idilbi, X.d. Ji, J.P. Ma and F. Yuan, Phys. Rev. D 73 (2006) 077501; A. Idilbi, X.d. Ji and F. Yuan, Nucl. Phys. B 753 (2006) 42; V. Ravindran, Nucl. Phys. B 746 (2006) 58; V. Ahrens, T. Becher, M. Neubert and L. L. Yang, Phys. Rev. D 79 (2009) 033013.

[8] S. Marzani, R.D. Ball, V. Del Duca, S. Forte and A. Vicini, Nucl. Phys. B 800 (2008) 127; R. V. Harlander and K. J. Ozeren, JHEP 0911 (2009) 088; A. Pak, M. Rogal and M. Steinhauser, arXiv:0911.4662 [hep-ph].

[9] A. Djouadi and P. Gambino, Phys. Rev. Lett. 73 (1994) 2528; A. Djouadi, P. Gambino and B. A. Kniehl, Nucl. Phys. B 523, 17 (1998).

[10] U. Aglietti, R. Bonciani, G. Degrassi and A. Vicini, Phys. Lett. B 595 (2004) 432; Phys. Lett. B 600 (2004) 57.

[11] G. Degrassi and F. Maltoni, Phys. Lett. B 600 (2004) 255.

[12] S. Actis, G. Passarino, C. Sturm and S. Uccirati, Nucl. Phys. B 811 (2009) 182.

[13] U. Aglietti, R. Bonciani, G. Degrassi and A. Vicini, arXiv:hep-ph/0610033; S. Actis, G. Passarino, C. Sturm and S. Uccirati, Phys. Lett. B 670 (2008) 12.

[14] M. Steinhauser, Phys. Rev. D 59 (1999) 054005.

[15] C. Anastasiou, R. Boughezal and F. Petriello, JHEP 0904 (2009) 003.

[16] S. Actis, G. Passarino, C. Sturm and S. Uccirati, Phys. Lett. B 669 (2008) 62.

[17] G. Passarino, C. Sturm and S. Uccirati, Phys. Lett. B 655 (2007) 298.

[18] A. Denner, S. Dittmaier, M. Roth and L.H. Wieders, Nucl. Phys. B 724 (2005) 247; A. Denner and S. Dittmaier, Nucl. Phys. Proc. Suppl. 160 (2006) 22; A. Denner, S. Dittmaier, M. Roth and D. Wackeroth, Nucl. Phys. B 560 (1999) 33.

[19] M. J. G. Veltman, Physica 29, 186 (1963).

[20] C. Mœller, K. Danske. Vid. Selsk. 22, no 19 (1946); R. Peierls, Proceedings of the 1954 Glasgow Conference on Nuclear and Meson Physics, (Pergamon Press, New York, 1955) p. 296; R. Jacob and R. G. Sachs, Phys. Rev. 121 (1961) 350.

[21] P. A. Grassi, B. A. Kniehl and A. Sirlin, Phys. Rev. Lett. 86 (2001) 389 [arXiv:hep-th/0005149]; B. A. Kniehl and A. Sirlin, Phys. Rev. Lett. 81 (1998) 1373; B. A. Kniehl and A. Sirlin, Phys. Rev. D 77, 116012 (2008); B. A. Kniehl and A. Sirlin, Phys. Lett. B 530, 129 (2002). 